\title{
Multipath Routing Algorithms for Congestion Minimization
}

\author{
Ron Banner, Senior Member, IEEE, and Ariel Orda, Fellow, IEEE
}

\begin{abstract}
Unlike traditional routing schemes that route all traffic along a single path, multipath routing strategies split the traffic among several paths in order to ease congestion. It has been widely recognized that multipath routing can be fundamentally more efficient than the traditional approach of routing along single paths. Yet, in contrast to the single-path routing approach, most studies in the context of multipath routing focused on heuristic methods. We demonstrate the significant advantage of optimal (or near optimal) solutions. Hence, we investigate multipath routing adopting a rigorous (theoretical) approach. We formalize problems that incorporate two major requirements of multipath routing. Then, we establish the intractability of these problems in terms of computational complexity. Finally, we establish efficient solutions with proven performance guarantees.
\end{abstract}

Index Terms-Computer networks, congestion avoidance, routing protocols.

\section{INTRODUCTION}

$\mathbf{C}$ URRENT routing schemes typically focus on discovering a single "optimal" path for routing, according to some desired metric. Accordingly, traffic is always routed over a single path, which often results in substantial waste of network resources. Multipath routing is an alternative approach that distributes the traffic among several "good" paths instead of routing all traffic along a single "best" path.

Multipath routing can be fundamentally more efficient than the currently used single-path routing protocols. It can significantly reduce congestion in "hot spots," by deviating traffic to unused network resources, thus, improving network utilization and providing load balancing [16]. Moreover, congested links usually result in poor performance and high variance. For such circumstances, multipath routing can offer steady and smooth data streams [6].

Multipath routing algorithms that optimally split traffic between a given set of paths have been investigated in the context of flow control (e.g., [14], [19], [20]). Yet, the selection of the routing paths is another major design consideration that has a drastic effect on the resulting performance. Therefore, although many flow-control algorithms are optimal for a given set of routing paths, their performance can significantly differ for different sets of paths. Accordingly, in this paper, we focus on multipath routing algorithms that both select the routing paths and split traffic among them.

Manuscript received August 2, 2005; revised February 22, 2006; approved by IEEE/ACM TRANSACTIONS ON NETWORKING Editor R. Srikant. The conference version of this paper appeared in Proceedings of the IFIP Networking, 2005.

The authors are with the Department of Electrical Engineering, TechnionIsrael Institute of Technology, Haifa 32000, Israel (e-mail: banner@tx.technion.ac.il; ariel@ee.technion.ac.il).

Digital Object Identifier 10.1109/TNET.2007.892850
Previous studies and proposals on multipath routing in the previous context have focused on heuristic methods. In [22], a multipath routing scheme, termed equal cost multipath (ECMP), has been proposed for balancing the load along multiple shortest paths using a simple round-robin distribution. By limiting itself to shortest paths, ECMP considerably reduces the load balancing capabilities of multipath routing; moreover, the equal partition of flows along the (shortest) paths (resulting from the round robin distribution) further limits the ability to decrease congestion through load balancing. OSPF-OMP [28] allows splitting traffic among paths unevenly; however, the traffic distribution mechanism is based on a heuristic scheme that often results in an inefficient flow distribution. Both [29] and [31] considered multipath routing as an optimization problem with an objective function that minimizes the congestion of the most utilized link in the network; however, they focused on heuristics and did not consider the quality of the selected paths. In [23], a scheme was presented to proportionally split traffic among several "widest" paths that are disjoint with respect to the bottleneck links. However, here too, the scheme is heuristic and evaluated by way of simulations.

Through comprehensive simulations, we show that multipath solutions obtained by optimal congestion reduction schemes are fundamentally more efficient than solutions obtained by heuristics. Specifically, we show that if the traffic distribution mechanism of the ECMP or OSPF-OMP schemes had been optimal, the network congestion would have decreased by a factor of more than 2.5; moreover, these simulations indicate that optimal traffic distribution mechanisms become significantly more efficient by just slightly alleviating the requirement to route along shortest paths. Hence, the full potential of multipath routing is far from having been exploited.

Accordingly, in this study, we investigate multipath routing adopting a rigorous approach, and formulate it as an optimization problem of minimizing network congestion. Under this framework, we consider two fundamental requirements. First, each of the chosen paths should usually be of satisfactory "quality." Indeed, while better load balancing is achieved by allowing the employment of paths other than shortest, paths that are substantially inferior (i.e., "longer") may be prohibited. Therefore, we consider the problem of congestion minimization through multipath routing subject to a restriction on the "quality" (i.e., length) of the chosen paths.

Another practical restriction is on the number of routing paths per destination, which is due to the following reasons [23]. First, establishing, maintaining, and tearing down paths pose considerable overhead. Second, the complexity of a scheme that distributes traffic among multiple paths considerably increases with the number of paths. Third, often there is a limit on the 
number of explicitly routing paths (such as label-switched paths in MPLS [26]) that can be set up between a pair of nodes. Therefore, in practice, it is desirable to use as few paths as possible while at the same time minimize the network congestion.

Link state protocols [15] constitute an important class of routing protocols that can be used for the implementation of multipath solutions. In this class of protocols, each node maintains a "map" of the network that enables it to compute the routes. When a network link changes status, a notification is flooded throughout the network and all nodes recompute their routes according to their updated maps. It has been noted [15] that link state protocols have the following desirable properties: 1) they can employ more complicated routing approaches and can compute more accurate routes than can be computed with distance vector protocols; 2) they respond faster to changes and impose less communication overhead than the alternative (distance vector) protocols; and 3) they are applicable for the Internet (e.g., both OSPF and IS-IS are link state protocols) and may be applicable for ad hoc networks (see, e.g., [25]). Accordingly, in this paper, we assume a link-state routing environment and formulate our problems accordingly.

Our Results: Consider first the problem of minimizing the congestion under the requirement to route traffic along paths of "satisfactory" quality. We first show that the considered problem is NP-hard, yet admits a pseudo-polynomial solution. Accordingly, we design two algorithms. The first is an optimal algorithm with a pseudo-polynomial running time and the second approximates the optimal solution to any desired degree of precision at the (proportional) cost of increasing its running time (i.e., an $\varepsilon$-optimal approximation scheme). In addition, we show that these algorithms can be extended to offer solutions to reliability related problems. Consider now the requirement of limiting the number of paths per destination. We show that minimizing the congestion under this restriction is NP-hard as well. However, we establish a computationally efficient two-approximation scheme for the problem, i.e., our algorithm provides a solution that, in terms of congestion, is within a factor of at most two away from the optimum.

Organization: In Section II, we introduce some terminology and definitions, and formulate the main problems considered in this study. In Section III, we consider the problem of minimizing congestion under path quality constraints and provide both accurate as well as approximate solutions. In Section IV, we investigate the problem of minimizing congestion subject to a restriction on the number of paths per destination; we show that the problem is NP-hard and provide a computationally efficient two-approximation scheme. In Section V, we present simulation results that demonstrate the major advantage of optimal congestion reduction schemes over two well-known heuristics. Finally, Section VI summarizes the main results and discusses future directions for future research.

\section{Model AND PROBlem Formulation}

A network is represented by a connected directed graph $G(V, E)$, where $V$ is the set of nodes and $E$ is the set of links. Let $N=|V|$ and $M=|E|$. A path is a finite sequence of nodes $p=\left(v_{0}, v_{1}, \ldots v_{h}\right)$, such that, for 0 $\leq n \leq h-1,\left(v_{n}, v_{n+1}\right) \in E$. A path is simple if all its nodes are distinct. Given a source node $s \in V$ and a target node $t \in V, P^{(s, t)}$ is the set of (all) directed paths in $G(V, E)$ from $s$ to $t$. Let $P_{\text {simple }}^{(s, t)} \subseteq P^{(s, t)}$ represent the set of (all) simple paths in $G(V, E)$ from $s$ to $t$. Finally, for each path $p \in P_{\text {simple }}^{(s, t)}$ and link $e \in E$, define a link-path indicator $\delta_{e}(p)$, which is 1 if link $e$ is contained in $p$, and is 0 otherwise.

We consider a link-state routing environment, where each source node has an image of the entire network. Each link $e \in E$ is assigned a length $l_{e} \in \mathbb{Z}^{+}$and a capacity $c_{e} \in \mathbb{Z}^{+}$. Given a (nonempty) path $p$, the length $L(p)$ of $p$ is defined as the sum of lengths of its links, namely, $L(p) \triangleq \sum_{e \in p} l_{e}$.

We consider two types of network flow representations. In the path flow representation, each variable $f_{p} \geq 0$ is the flow on some simple path $p \in P_{\text {simple. }}^{(s, t)}$ Given two nodes $s, t \in V$ and a (flow) demand $\gamma$, we say that a path flow $f$ is feasible iff it satisfies the flow demand requirement, i.e., $\sum_{p \in P_{\text {Simple }}^{(s, t)}} f_{p}=\gamma$, and the capacity constraints, i.e., $\sum_{p \in P_{\text {Simple }}^{(s, t)}} \delta_{e}(p) \cdot f_{p} \leq c_{e}$ for each $e \in E$. In the link flow representation, each variable $f_{e} \geq 0$ is the flow on some link $e \in E$. Given two nodes $s, t \in V$ and a demand $\gamma$, a link flow $f$ is feasible iff there exists some feasible path flow $\tilde{f}$ for the given instance such that $f_{e}=\sum_{p \in P_{\text {Simple }}^{(s, t)}} \delta_{e}(p) \cdot \tilde{f}_{p}$ for each $e \in E$.

We proceed to formulate the criterion for congestion. Given a network $G(V, E)$ and a link flow $\left\{f_{e}\right\}$, the value $\frac{f_{e}}{c_{e}}$ is the link congestion factor and the value $\max _{e \in E}\left\{\frac{f_{e}}{c_{e}}\right\}$ is the network congestion factor. As noted in [2], [16], and [29], the network congestion factor provides a good indication of congestion. In [4], we show that the problem of minimizing the network congestion factor is equivalent to the well-known maximum flow problem [1]. Hence, when there are no restrictions on the paths (in terms of the number of paths or the length of each path), one can find a path flow that minimizes the network congestion factor in polynomial time through a standard max-flow algorithm.

We are ready to formulate the two problems considered in this study. The first problem aims at minimizing the network congestion factor subject to a restriction on the "quality" (i.e., length) of each of the chosen paths.

Problem RMP (Restricted Multipath): Consider a network $G(V, E)$, two nodes $s, t \in V$, a length $l_{e}>0$, and a capacity $c_{e}>0$ for each link $e \in E$, a demand $\gamma>0$, and a length restriction $L$ for each routing path. Find a feasible path flow that minimizes the network congestion factor such that, if $P \subseteq$ $P^{(s, t)}$ is the set of paths in $P^{(s, t)}$ that are assigned a positive flow, then, for each $p \in P$, it holds that $L(p) \leq L$.

Remark 1: For convenience, and without loss of generality, we assume that the length $l_{e}$ of each link $e \in E$ is not larger than the length restriction $L$. Clearly, links that are longer than $L$ can be erased.

The next problem considers the requirement to limit the number of different paths over which a given demand is shipped while at the same time minimizing the network congestion factor.

Problem KPR (K-Path Routing): Given are a network $G(V, E)$, two nodes $s, t \in V$, a capacity $c_{e}>0$ for each link 
$e \in E$, a demand $\gamma>0$, and a restriction on the number of routing paths $K$. Find a feasible path flow that minimizes the network congestion factor, such that, if $P \subseteq P^{(s, t)}$ is the set of paths in $P^{(s, t)}$ that are assigned a positive flow, then $|P| \leq K$.

Remark 2: In both problems, the source destination pair $(s, t)$ is assumed to be connected, i.e., $\left|P^{(s, t)}\right| \geq 1$.

Remark 3: In both problem formulations, it is possible to limit the link congestion factor $\frac{f_{e}}{c_{e}}$ of each $e \in E$ to any desired congestion level $\alpha_{e}$ by replacing the given capacity value $c_{e}$ with a new capacity value $\alpha_{e} \cdot c_{e}$. Clearly, the capacity constraint $f_{e} \leq \alpha_{e} \cdot c_{e}$ (that both problems must satisfy) assures that the link congestion factor would be at most $\alpha_{e}$.

\section{Minimizing CONGESTION UNDER PATH QUALITY CONSTRAINTS}

In this section, we investigate Problem RMP, i.e., the problem of minimizing congestion under path quality constraints. In Section III-A, we prove that Problem RMP is computational intractable. Accordingly, in Section III-B, we establish a pseudo-polynomial solution and in Section III-C we design an $\varepsilon$-optimal approximation scheme for the problem.

\section{A. Intractability of Problem RMP}

We show that Problem RMP can be reduced to the Partition Problem [12].

Theorem 1-Problem RMP is NP-hard:

Proof: Consider the following instance of the Partition problem; given a set of elements $a_{1}, a_{2}, \ldots, a_{2 n}$ that constitute a set $A$ with size $s(a) \in \mathbb{Z}^{+}$for each $a \in A$, find a subset $A^{\prime} \subseteq A$ such that $A^{\prime}$ contains exactly one element of $a_{2 i-1}, a_{2 i}$ for every $1 \leq i \leq n$ and $\sum_{a \in A^{\prime}} s(a)=\sum_{a \in A-A^{\prime}} s(a)$.

We transform Partition to RMP as follows (see also Fig. 1).

1) Given an element $a_{i} \in A$ with size $s\left(a_{i}\right)$, define a unit capacity link $u_{i} \rightarrow v_{i}$ with length $s\left(a_{i}\right)$.

2) For each link $u_{2 i-1} \rightarrow v_{2 i-1}, 1 \leq i \leq n$, define a link $v_{2 i-1} \rightarrow u_{2 i+1}$ and a link $v_{2 i-1} \rightarrow u_{2 i+2}$. Assign to both a unit capacity and a zero length.

3) For each link $u_{2 i} \rightarrow v_{2 i}, 1 \leq i \leq n$, define a link $v_{2 i} \rightarrow u_{2 i+1}$ and a link $v_{2 i} \rightarrow u_{2 i+2}$. Assign to both a unit capacity and a zero length.

4) Define links $s \rightarrow u_{1}, s \rightarrow u_{2}$ and links $v_{2 n-1} \rightarrow t, v_{2 n} \rightarrow$ $t$. Assign to each a unit capacity and a zero length.

5) Set: $L \leftarrow \frac{1}{2} \cdot \sum_{a \in A} s(a)$ and $\gamma \leftarrow 2$.

We shall prove that it is possible to transfer two flow units over paths whose lengths are not larger than $L$ without exceeding a network congestion factor of $\alpha=1$ iff there is a subset $A^{\prime} \subseteq A$ such that $A^{\prime}$ contains exactly one element of $a_{2 i-1}, a_{2 i}$ for every $1 \leq i \leq n$ and $\sum_{a \in A^{\prime}} s(a)=\sum_{a \in A \backslash A^{\prime}} s(a)$. (Remark: We refer to elements and their sizes interchangeably. $\Leftarrow$ : Suppose there exists a subset $A^{\prime} \subseteq A$ such that $A^{\prime}$ contains exactly one of $a_{2 i-1}, a_{2 i}$ for each $1 \leq i \leq n$ and $\sum_{a \in A^{\prime}} s(a)=\sum_{a \in A \backslash A^{\prime}} s(a)$. Then, it is easy to see that the selection of the links that represents the elements in $A^{\prime}$ and the zero length links that connect those links constitutes a path. Also, it is easy to see that this path is disjoint to the path that the complement subset $A \backslash A^{\prime}$ defines. Since all capacities are equal to 1 , we have two disjoint paths that can transfer together exactly two units of flow without violating the congestion

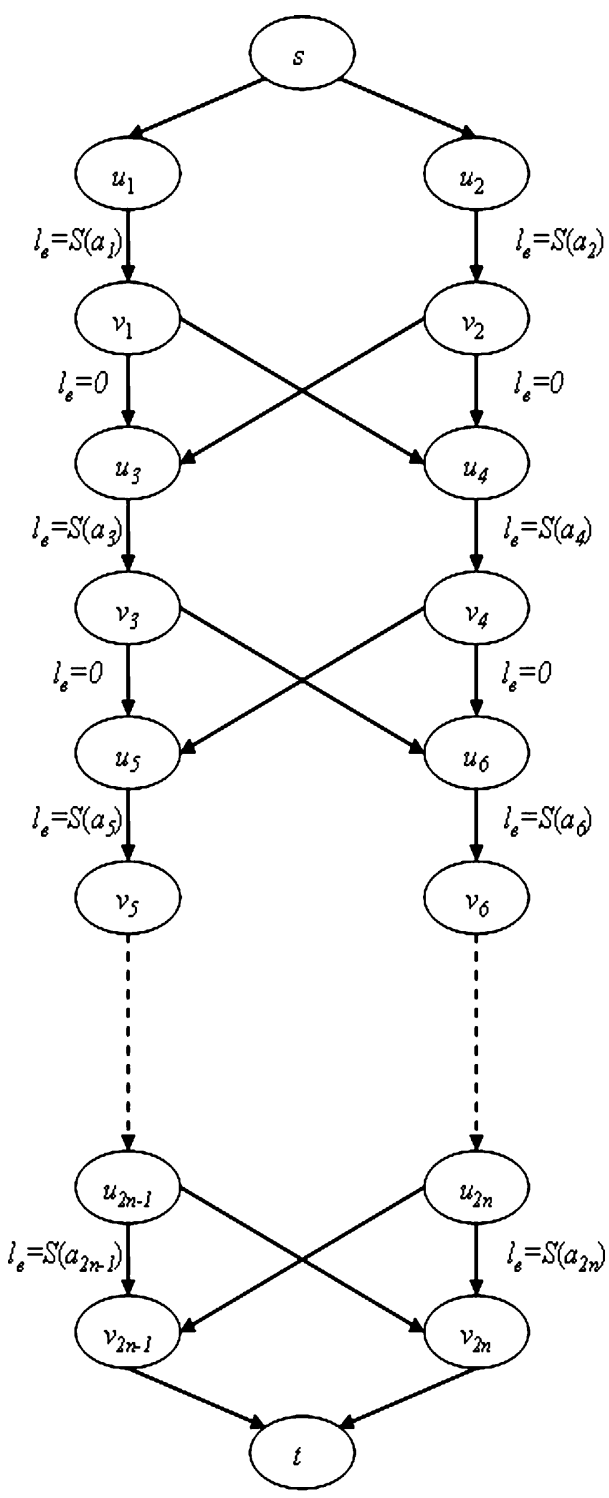

Fig. 1. Reduction of partition to RMP.

constraint $\alpha=1$. The length restriction is preserved since the two defined paths have length of $0.5 \cdot \sum_{a \in A} s(a)$, which was defined to be the length restriction $L . \Rightarrow$ :Suppose there is a path flow that transfers two flow units over paths that are not longer than $L$. Select one path that transfers a positive flow and denote it as $p$. Define an empty set $S$. For every link in $p$, with length $s\left(a_{i}\right)$, insert the element $a_{i}$ into $S$. Since all links in the graph have one unit of capacity, the selected path $p$ is not able to transfer more than one unit of flow. Now, delete all the links that constitute path $p$. Since $p$ transfers at most one unit of flow, there must be another path that is disjoint to the selected path and transfers a positive flow over the links that were left in the graph. For each link in that path with size $s\left(a_{i}\right)$, insert the element $a_{i}$ into a different set $S^{\prime}$. We will now prove that $A=S \cup S^{\prime}, S \cap S^{\prime}=\emptyset$, and, finally, $\sum_{a \in S} s(a)=\sum_{a \in S^{\prime}} s(a)$.

Since $S$ and $S^{\prime}$ were constructed out of disjoint paths, it is obvious that $S \cup S^{\prime}=\emptyset$. Since every path must traverse either $s\left(a_{2 i-1}\right)$ or $s\left(a_{2 i}\right)$ for each $1 \leq i \leq n$, and since both paths are 


\begin{tabular}{|c|c|c|}
\hline \multicolumn{3}{|c|}{ Program RMP $\left(G(V, E),\{s, t\},\left\{l_{e}\right\},\left\{c_{e}\right\}, \gamma, L\right)$} \\
\hline Minimize $\alpha$ & & (1) \\
\hline \multicolumn{3}{|l|}{ Subject to: } \\
\hline$\sum_{e \in O(v)} f_{e}^{\lambda}-\sum_{e \in I(v)} f_{e}^{\lambda-l_{e}}=0$ & $\forall v \in V \backslash\{s, t\}, \forall \lambda \in[0, L]$ & (2) \\
\hline$\sum_{e \in O(s)} f_{e}^{\lambda}-\sum_{e \in I(s)} f_{e}^{\lambda-l_{e}}=0$ & $\forall \lambda \in[1, L]$ & (3) \\
\hline$\sum_{e \in O(s)} f_{e}^{0}=\gamma$ & & (4) \\
\hline$\sum_{\lambda=0}^{L} f_{e}^{\lambda} \leq c_{e} \cdot \alpha$ & $\forall e \in E$ & (5) \\
\hline$f_{e}^{\lambda}=0$ & $\forall e \in E, \lambda \notin\left[0, L-l_{e}\right]$ & (6) \\
\hline$f_{e}^{\lambda} \geq 0$ & $\forall e \in E, \lambda \in[0, L]$ & (7) \\
\hline$\alpha \geq 0$ & & (8) \\
\hline
\end{tabular}

Fig. 2. Program RMP.

disjoint, $S \cup S^{\prime}=\left\{a_{i}\right\}_{i=1}^{2 n}=A$. Finally, since both paths have lengths that are not longer than $L$, we have

$$
\sum_{a \in S} s(a) \leq L, \quad \sum_{a \in S^{\prime}} s(a) \leq L .
$$

Since $L \triangleq \frac{1}{2} \cdot \sum_{a \in A} s(a)$ and $S \cup S^{\prime}=A$, we get

$$
\sum_{a \in S} s(a)+\sum_{a \in S^{\prime}} s(a)=\sum_{a \in A} s(a)=2 \cdot L .
$$

Note that if variables $x_{1}, x_{2}$ satisfy $x_{1} \leq B, x_{2} \leq B$ and in addition $x_{1}+x_{2}=2 \cdot B$, it follows that $x_{1}=x_{2}=B$. Accordingly, we conclude from (1) and (2) that $\sum_{a \in S} s(a)=$ $\sum_{a \in S^{\prime}} s(a)=L$.

Thus, problem RMP is NP hard.

\section{B. Pseudo-Polynomial Algorithm for Problem RMP}

The first step towards obtaining a solution to Problem RMP is to define it as a linear program. To that end, we need some additional notation.

Recall that we are given a network $G(V, E)$, two nodes $s, t \in$ $V$, a length $l_{e}>0$, and a capacity $c_{e}>0$ for each link $e \in E$, a demand $\gamma>0$ and a length restriction $L$ for each routing path. Let $\alpha$ be the network congestion factor. Denote by $f_{e}^{\lambda}$ the total flow along $e=(u, v) \in E$ that has been routed from $s$ to $u$ through paths with a total length $\lambda$. Finally, for each $v \in$ $V$, denote by $O(v)$ the set of links that emanate from $v$, and by $I(v)$ the set of links that enter that node, namely, $O(v)=$ $\{(v, l) \mid(v, l) \in E\}$ and $I(v)=\{(w, v) \mid(w, v) \in E\}$. Then, Problem RMP can be formulated as a linear program over the variables $\left\{\left\{f_{e}^{\lambda}\right\}, \alpha\right\}$, as specified in Fig. 2 .

The objective function (1) minimizes the network congestion factor. Constraints (2), (3), and (4) are nodal flow conservation constraints. Equation (2) states that the traffic flowing out of node $v$, which has traversed through paths $p \in P^{(s, v)}$ of length $L(p)=\lambda$, has to be equal to the traffic flowing into node $v$, through paths $p^{\prime} \in P^{(s, u)}$ and links $e=(u, v) \in E$, such that $L\left(p^{\prime}\right)+l_{e}=\lambda$; since $\lambda \in[0, L]$, the length restriction is obeyed; finally, (2) must be satisfied for each node other than the source $s$ and the target $t$. Equation (3) extends the validity

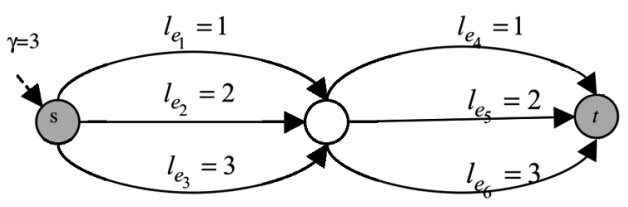

Fig. 3. Single link flow can be decomposed into several path flows. Some of them satisfy the length restriction and the rest violate it.

of (2) to hold for traffic that encounters source $s$ after it has already passed through paths with non-zero length. Informally, (3) states that "old" traffic that emanates from $s$ not for the first time (through a directed cycle that contains the source $s$ ) must satisfy the nodal flow conservation constraint of (2), which solely focuses on nodes from $V \backslash\{s, t\}$. Equation (4) states that the total traffic flowing out of source $s$, which has traversed paths of length $L=0$, must be equal to the demand $\gamma$. Informally, (4) states that the total "new" traffic that emanates from the source $s$ for the first time must satisfy the flow demand $\gamma$. Equation (5) is the link capacity utilization constraint. It states that the maximum link utilization is not larger than the value of the variable $\alpha$, i.e., the network congestion factor is at most $\alpha$. Expression (6) rules out nonfeasible flows and Expressions (7) and (8) restrict all variables to be nonnegative.

We can solve Program RMP (Fig. 1) using any polynomial time algorithm for linear programming [18]. The solution to the problem is then achieved by decomposing the output of Program RMP (i.e., link flow $\left\{f_{e}^{\lambda}\right\}$ ) into a path flow that satisfies the length restriction $L$. Standard flow techniques that transform flows along links into flows along paths (e.g., the flow decomposition algorithm [1]), cannot be used for our purpose since they do not respect the length restrictions. This is illustrated by the following example.

Example 1: Consider the network depicted in Fig. 3. Suppose that the flow along each link is equal to one unit, i.e., $f_{e_{i}}=1$ for each $i \in[1,6]$. Moreover, assume that the length restriction is 4 . There are several path flows that can be decomposed out of the link flow $\left\{f_{e}\right\}$. For example, one such is the path flow $f_{1}$ that assigns one unit of flow to each of the paths $\left\{e_{1}, e_{4}\right\},\left\{e_{2}, e_{5}\right\},\left\{e_{3}, e_{6}\right\}$; indeed, since $f_{1}$ transfers one unit of flow along each link, its link flow representation is $\left\{f_{e}\right\}$. However, since the length of the path $\left\{e_{3}, e_{6}\right\}$ is 6 , the path flow $f_{1}$ violates the length restriction. On the other hand, if we decompose the link flow $\left\{f_{e}\right\}$ into the path flow that assigns one unit to each of the paths $\left\{e_{1}, e_{6}\right\},\left\{e_{2}, e_{5}\right\},\left\{e_{3}, e_{4}\right\}$, the length restriction is satisfied on all paths.

Our goal is, therefore, to establish an efficient algorithm that decomposes link flow $\left\{f_{e}^{\lambda}\right\}$, into a path flow that satisfies the length restrictions. Accordingly, consider Algorithm PFC, which is specified in Fig. 4. This algorithm is an iterative algorithm that identifies at each iteration a single path with a length of at most $L$ whose corresponding link flows $\left\{f_{e}^{\lambda}\right\}$ are all positive; the path is identified through procedure path construction, which is specified in Fig. 5. The flow over the corresponding path is defined to be equal to the smallest flow $f_{e}^{\lambda}$ that belongs to the path. Then, the algorithm subtracts the flow that traverses through that path from the demand $\gamma$ and from each variable $f_{e}^{\lambda}$ in the path. The (iterative) algorithm repeats this process until 


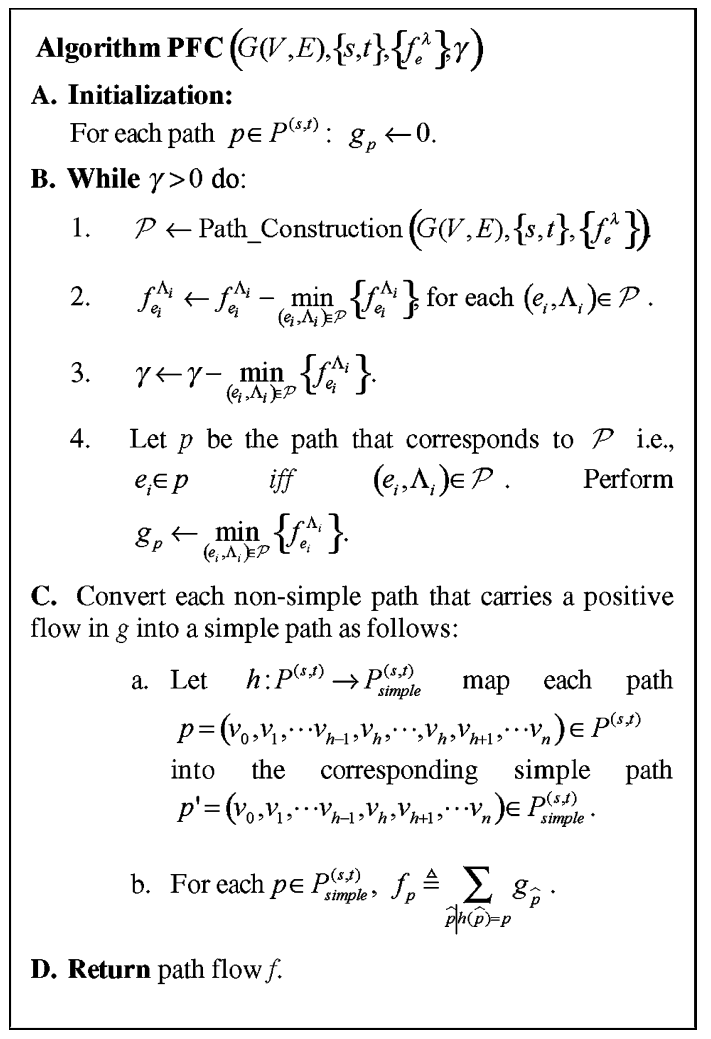

Fig. 4. Algorithm PFC.

Procedure Path_Construction $\left(G(V, E),\{s, t\},\left\{f_{e}^{\lambda}\right\}\right)$
Initialization
$\quad \mathcal{P} \leftarrow \phi, u_{0} \leftarrow s, \Lambda_{0} \leftarrow 0, i \leftarrow 0$
While $u_{i} \neq t$ do
1. Select a positive variable $f_{e_{i}}^{\Lambda_{i}}$ such that
$\quad e_{i} \triangleq\left(u_{i}, u_{i+1}\right) \in E$.
2. $\quad \mathcal{P} \leftarrow \mathcal{P} \cup\left\{\left(e_{i}, \Lambda_{i}\right)\right\}, \Lambda_{i+1} \leftarrow \Lambda_{i}+l_{e_{i}}, i \leftarrow i+1$.
Return $\mathcal{P}$

Fig. 5. Procedure path construction.

the demand $\gamma$ is zeroed. Thus, the resulting path flow transfers $\gamma$ flow units along paths with length of at most $L$. Finally, since procedure path construction might return nonsimple paths, the algorithm converts all nonsimple paths in the resultant path flow into simple paths by eliminating their loops.

We turn to explain the main idea behind procedure path construction (Fig. 5). The procedure identifies a path $p \in P^{(s, t)}, p: s \stackrel{e_{0}}{\rightarrow} u_{1} \stackrel{e_{1}}{\rightarrow} u_{2}, \ldots, u_{h-1} \stackrel{e_{h-1}}{\rightarrow} t$, whose corresponding variables $\left\{f_{e_{0}}^{0}, f_{e_{1}}^{l_{e_{0}}}, \ldots, f_{e_{h-1}}^{l_{e_{0}}+\cdots+l_{e_{h-2}}}\right\}$ are all positive. Consider the positive variable $f_{e_{h-1}}^{l_{e_{0}}+\cdots+l_{e_{h-2}}}$. Since (6) of Program RMP zeroes each variable $f_{e}^{\lambda}$ with a length $\lambda \notin\left[0, L-l_{e}\right]$, it holds for the positive variable $f_{e_{h-1}}^{l_{e_{0}}+\cdots+l_{e_{h-2}}}$ that $l_{e_{0}}+l_{e_{1}}+\cdots+l_{e_{h-2}} \leq L-l_{e_{h-1}}$; hence, $\sum_{i=0}^{h-1} l_{e_{i}} \leq L$. In other words, each path $p \in P^{(s, t)}$ with a corresponding sequence of positive variables $\left\{f_{e_{0}}^{0}, f_{e_{1}}^{l_{e_{0}}}, \ldots, f_{e_{h-1}}^{l_{e_{0}}+\cdots+l_{e_{h-2}}}\right\}$ has a length $L(p), L(p)=\sum_{i=0}^{h-1} l_{e_{i}} \leq L$. Thus, in order to establish a path $p \in P^{(s, t)}$ with a length of at most $L$, it is sufficient to find a sequence of positive variables $\left\{f_{e_{0}}^{0}, f_{e_{1}}^{l_{e_{0}}}, \ldots, f_{e_{h-1}}^{l_{e_{0}}+\cdots+l_{e_{h-2}}}\right\}$ such that the link $e_{0}$ emanates from source $s$ and the link $e_{h-1}$ enters into the destination $t$, i.e., $e_{0} \in O(s)$ and $e_{h-1} \in I(t)$. To that end, we employ the following property that characterizes the solutions of Program RMP. If a positive flow $f_{e}^{\lambda-l_{e}}$ enters through the link $e=(u, v)$ into the node $v \in V \backslash\{s, t\}$, it follows that there is some link $e^{\prime}=(v, w)$ such that $f_{e^{\prime}}^{\lambda}>0$. Therefore, since $f_{e^{\prime \prime}}^{0}>0$ for some link $e^{\prime \prime}$; that emanates from the source $s$, it is possible to follow positive flows (variables) from $s$ in order to construct a sequence of positive variables $\left\{f_{e_{0}}^{0}, f_{e_{1}}^{l_{e_{0}}}, \ldots, f_{e_{k}}^{l_{e_{0}}+\cdots+l_{e_{k-1}}}, \ldots\right\}$ such that $e_{0} \in O(s)$. We now prove that, by following these positive variables for a finite number of times, we eventually encounter a positive variable $f_{e_{k}}^{l_{e_{0}}+\cdots+l_{e_{k}}}$ such that $e_{k}$ enters the destination $t$, i.e., we eventually identify a directed path from $s$ to $t$ with a length of at most $L$.

Lemma 1: Consider the nodes $\left\{u_{i}\right\}$ identified by procedure path construction (step 1). There exists an $h, h \leq L$, such that the sequence $\left(u_{0}, u_{1}, \ldots, u_{h}\right)$ is a path from $s$ to $t$.

Proof: It follows from constraint (4) that, since $\gamma>0$, there exists some link $e_{0}=\left(u_{0}, u_{1}\right)$ such that the variable $f_{e_{0}}^{0}$ is positive. Then, from constraints (2) and (3), it follows that, if $u_{1} \neq t$, then there exists some link $e_{1}=\left(u_{1}, u_{2}\right)$ such that the variable $f_{e_{1}}^{l_{e}}$ is positive. Thus, applying constraints (2) and (3) for any index $i$, it follows that, if there exists a positive variable $f_{e_{i}}^{\Lambda_{i}}$, where $\Lambda_{i} \triangleq \sum_{k=0}^{i-1} l_{e_{k}}$, then, unless $u_{i+1}=t$, there exists a link $e_{i+1}=\left(u_{i+1}, u_{i+2}\right)$ such that the variable $f_{e_{i+1}}^{\Lambda_{i}+l_{e_{i}}}$ is positive. Therefore, if $f_{e_{i}}^{\Lambda_{i}}=0$ for each $i>K$, it must hold that there exists an index $j, j \leq K$, such that $u_{j}=t$. Hence, in order to establish the lemma, it is sufficient to show that $f_{e_{i}}^{\Lambda_{i}}=0$ for each $i>L$.

Indeed, since $l_{e} \in \mathbb{Z}^{+}$for each $e \in E$, it follows that $\Lambda_{i+1}-$ $\Lambda_{i} \geq 1$ for any index $i$; hence, $\Lambda_{i}>L$ for each $i>L$. Therefore, since it follows from constraint (6) that $f_{e_{i}}^{\Lambda_{i}}=0$ for each $\Lambda_{i}>L$, it holds that $f_{e_{i}}^{\Lambda_{i}}=0$ for each $i>L$.

For completion, in Fig. 6, we specify Algorithm RMP, which solves Problem RMP.

Next, we consider the complexity of Algorithm RMP. First, it follows from [18] that the complexity incurred by solving the linear program of step 1 is polynomial both in the number of variables $\left\{f_{e}^{\lambda}\right\}$ and in the number of constraints needed to formulate Program RMP. Thus, since both of these numbers are in the order of $M \cdot L$, the complexity of step 1 is polynomial in $O(M \cdot L)$. Consider now the complexity incurred by step 2 (Algorithm PFC). Since, by construction, each iteration of Algorithm PFC zeroes at least one variable $f_{e}^{\lambda}$, it follows that Algorithm PFC iterates for no more than the number of variables $\left\{f_{e}^{\lambda}\right\}$. Moreover, since the complexity of each iteration is dominated by the complexity of procedure path construction, which, according to Lemma 1 , consumes $O(L)$ operations, the complexity of Algorithm PFC is $L \cdot\left|\left\{f_{e}^{\lambda}\right\}\right|=O\left(M \cdot L^{2}\right)$. Thus, we conclude that the overall complexity of Algorithm RMP is polynomial in $M \cdot L^{2}$, i.e., Algorithm RMP is a pseudo-polynomial time algorithm [12]. Whenever the value of $L$ is polynomial in the size of the problem, Algorithm RMP is a poly- 


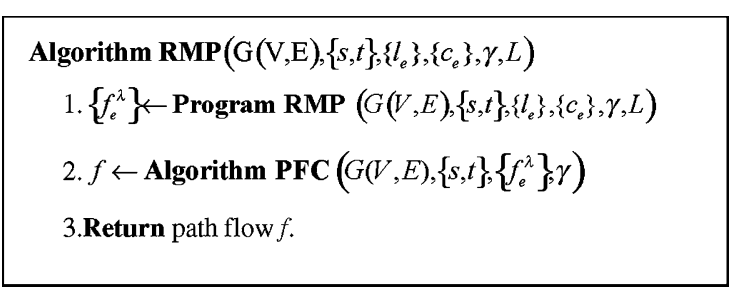

Fig. 6. Algorithm RMP.

nomial optimal algorithm for Problem RMP. One such case is when the hop-count metric is considered (i.e., $l_{e} \equiv 1$ ), since then $L \leq N-1$.

\section{C. $\varepsilon$-Optimal Approximation Scheme for Problem RMP}

In Section III-B, we established an optimal polynomial solution to Problem RMP for the case where the length restrictions are sufficiently small. In this section, we turn to consider the solution to Problem RMP for arbitrary length restrictions. As Theorem 1 establishes that Problem RMP is NP-hard for this general case, we focus on the design of an efficient algorithm that approximates the optimal solution.

Our main result is the establishment of an $\varepsilon$-optimal approximation scheme, which is termed the RMP approximation scheme. This scheme is based on Algorithm RMP, specified in Section III-B, which was shown to have a complexity that is polynomial in $M \cdot L^{2}$. Given an instance of Problem RMP and an approximation parameter $\varepsilon$, the RMP approximation scheme reduces the complexity of Algorithm RMP by first scaling down the length restriction $L$ by a factor $\Delta=\frac{L \cdot \varepsilon}{N}$ and then rounding it into an integer. Obviously, as a result, it must also scale down the length of each link. However, in order to ensure that the optimal network congestion factor does not increase, the RMP approximation scheme relaxes the constraints of the new instance with respect to the constraints of the original instance. Specifically, after the RMP approximation scheme scales down the length restriction and the length of each link by the factor $\Delta$, it rounds up the length restriction and rounds down the length of each link. Then, it invokes Algorithm RMP over the new instance, in order to construct a path flow that minimizes congestion while satisfying the relaxed length restrictions. In Theorem 2, we establish that the resulting path flow violates the length restriction by a factor of at most $(1+\varepsilon)$ and has a network congestion factor that is not larger than the optimal network congestion factor. The RMP approximation scheme is specified in Fig. 7.

Theorem 2: Given an instance $\left\langle G,\{s, t\}\left\{c_{e}\right\},\left\{l_{e}\right\}, \gamma L\right\rangle$ of problem RMP and an approximation parameter $\varepsilon$, the $R M P$ approximation scheme has a complexity that is polynomial in $1 / \varepsilon$ and in the size of the network. Moreover, the output of the scheme is a path flow $f$ that satisfies the following.

1) $\sum_{p \in P(s, t)} f_{p}=\gamma$, i.e., the flow demand requirement is satisfied.

2) If $\alpha^{*}$ is the network congestion factor of the optimal solution, then, for each e $\in E$, it holds that $\sum_{p \in P^{(s, t)}} \delta_{e}(p) \cdot f_{p} \leq \alpha^{*} \cdot c_{e}$, i.e., the network congestion factor is at most $\alpha^{*}$.

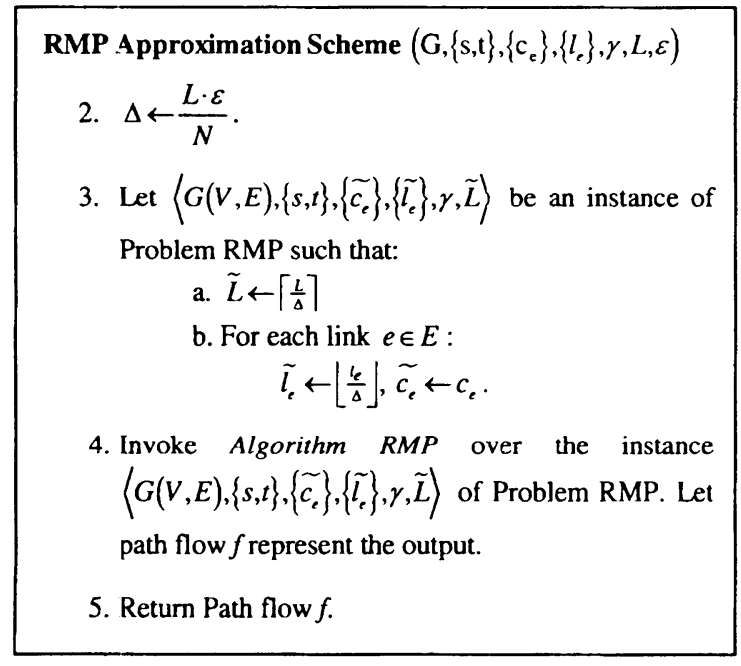

Fig. 7. RMP approximation scheme.

3) For each path $p \in P^{(s, t)}$, if $f_{p}>0$ then $p$ is simple and $L(p) \leq(1+\varepsilon) \cdot L$, i.e., the length restriction is violated by a factor of at most $(1+\varepsilon)$.

Proof: We first show that the complexity of the RMP approximation scheme is polynomial in $1 / \varepsilon$ and in the size of the network. To that end, recall that we have shown in Section III-B that the complexity incurred by invoking Algorithm RMP over any instance (of Problem RMP) with a length restriction $L$ is polynomial in $M \cdot L^{2}$. Therefore, since the RMP approximation scheme invokes Algorithm RMP over an instance with a length restriction $\tilde{L}=\left\lceil\frac{L}{\Delta}\right\rceil \leq \frac{N}{\varepsilon}+1$, it follows that its complexity is polynomial in $O\left(M \cdot\left(\frac{N}{\varepsilon}\right)^{2}\right)$. We turn to prove parts (1), (2), and (3) of the Theorem.

1) Since (4) in Program RMP specifies that $\sum_{e \in O(s)} f_{e}^{0}=\gamma$, it follows that every path flow representation $\left\{f_{p}\right\}$ of the link flow $\left\{f_{e}^{\lambda}\right\}$ must satisfy that $\sum_{p \in P^{(s, t)}} f_{p}=\gamma$.

2) Let $\alpha$ be the network congestion factor of the output $f$. We have to show that $\alpha \leq \alpha^{*}$. In Section III-B, we have shown that Algorithm RMP returns an optimal solution to Problem RMP. Thus, we only have to show that the space of feasible solutions of the instance $\left\langle G,\{s, t\},\left\{\tilde{c_{e}}\right\},\left\{\tilde{l_{e}}\right\}, \gamma, \tilde{L}\right\rangle$ (that constitutes an input to Algorithm RMP in step 4) contains the space of feasible solutions of the given instance $\left\langle G,\{s, t\},\left\{c_{e}\right\},\left\{l_{e}\right\}, \gamma, L\right\rangle$. Hence, it is sufficient to show that the length restrictions in $\left\langle G,\{s, t\},\left\{\tilde{c}_{e}\right\},\left\{\tilde{l_{e}}\right\}, \gamma, \tilde{L}\right\rangle$ are relaxed with respect to the length restrictions in $\left\langle G,\{s, t\},\left\{c_{e}\right\},\left\{l_{e}\right\}, \gamma, L\right\rangle$. Specifically, given a path $p$ in $G(V, E)$ that satisfy $L(p) \leq L$ with respect to $\left\{l_{e}\right\}$, we now show that $L(p) \leq \tilde{L}$ with respect to $\left\{l_{e}\right\}$. By the selection of $p$ it holds that $\sum_{e \in p} l_{e}=L(p) \leq L$; hence, $\sum_{e \in p} \frac{l_{e}}{\Delta} \leq \frac{L}{\Delta}$. Therefore, it holds that $\sum_{e \in p}\left\lfloor\frac{l_{e}}{\Delta}\right\rfloor \leq\left\lceil\frac{L}{\Delta}\right\rceil$; hence, by definition, $\sum_{e \in p} \tilde{l_{e}} \leq \tilde{L}$.

3) First, note that, by construction, the output of Algorithm RMP carries positive flow only over simple paths; hence, $p$ is simple. We turn to show that $L(p) \leq(1+\varepsilon) \cdot L$. Since $p$ transfers a positive flow, it holds that $\sum_{e \in p} \tilde{l_{e}} \leq$ $\tilde{L}$; hence, by definition $\sum_{e \in p}\left\lfloor\frac{l_{e}}{\Delta}\right\rfloor \leq\left\lceil\frac{L}{\Delta}\right\rceil$. Therefore, it 
holds that $\sum_{e \in p}\left(\frac{l_{e}}{\Delta}-1\right) \leq \frac{L}{\Delta}+1$; hence, $\sum_{e \in p} l_{e} \leq$ $L+(|p|+1) \cdot \Delta$, where $|p|$ is the hop count of path $p$. Finally, since $p$ is simple, it holds that $|p| \leq N-1$; hence, $L(p)=\sum_{e \in p} l_{e} \leq L+N \cdot \Delta==L+L \cdot \varepsilon=L \cdot(1+\varepsilon)$. Thus, the Theorem is established.

\section{Extensions}

In the following, we outline two important extensions to Problem RMP.

Multicommodity Extensions: In order to simplify the presentation of the solution to Problem RMP, we focused, in this paper, only on the single commodity case, i.e., we assume that only one source destination pair exists in every instance of Problem RMP. Following basically the same lines as in Sections III-A and III-B, we present in [4] a pseudo-polynomial solution and an $\varepsilon$-optimal approximation for the multicommodity extension of Problem RMP.

End-to-End Reliability Constraints: When traffic is split among multiple paths, a failure in any of these paths may result in the failure of the entire transmission. As a result, multipath routing may be more sensitive to network failures than single-path routing. One solution is to assign to each link $e \in E$ a failure probability $p_{e}$ (which can be estimated out of available failure statistics of each network component) and then to minimize the network congestion factor such that the traffic is routed solely along paths with a success probability larger than some given lower bound $\Pi$. Based on Algorithm RMP, it is possible to construct an $\varepsilon$-optimal approximation scheme for such a problem. Specifically, for any approximation parameter $\varepsilon$, the $\varepsilon$-optimal approximation scheme constructs an instance of Problem RMP by assigning a length $l_{e}=\left\lfloor\log _{1+\frac{\varepsilon}{N}}\left(1-p_{e}\right)\right\rfloor$ to each link $e$ and a length restriction $L=\left\lceil\log _{1+\frac{\varepsilon}{N}} \Pi\right\rceil$; then, it invokes Algorithm RMP over the resulting instance. In [4], we show that the solution is obtained within a time that is polynomial in $\frac{1}{\varepsilon}$; moreover, it is shown to minimize the network congestion factor while violating the requirement on the end-to-end success probability by a factor of at most $(1+\varepsilon)$. Due to space limits, the details are omitted here.

\section{Minimizing Congestion With $K$ Routing Paths}

In this section, we investigate Problem KPR, which minimizes congestion while routing traffic along at most $K$ different paths. In Section IV-A, we prove that Problem KPR is NP-hard in the general case but admits a (straightforward) polynomial solution when the restriction on the number of paths is larger than the number of links (i.e., $K \geq M$ ). Accordingly, in Sections IV-B and IV-C, we devise a two-approximation scheme for the more interesting case, where $K<M$.

\section{A. (In)tractability of Problem KPR}

The following Theorem establishes the intractability of Problem KPR for the general case.

Theorem 3-Problem KPR is NP-hard:

Proof: We reduce Problem KPR to the single-source unsplittable flow problem that was shown to be NP hard in [21]

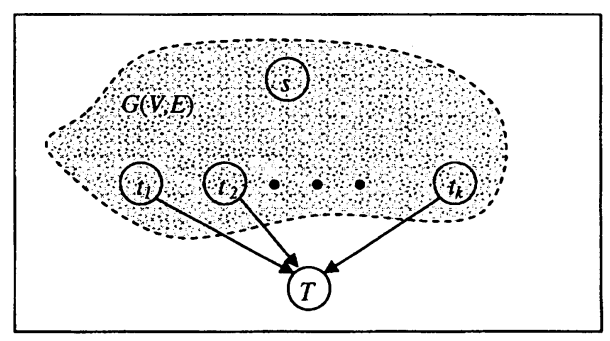

Fig. 8. Reducing the single-source unsplittable flow problem into Problem KPR.

and is defined as follows: given are a network $G(V, E)$, a capacity $c_{e}>0$ for each link $e \in E$, a set of source-destination pairs $\left(s, t_{1}\right),\left(s, t_{2}\right), \ldots,\left(s, t_{k}\right)$ associated with demands $\gamma_{1}, \gamma_{2}, \ldots, \gamma_{\kappa}$; is there an assignment of traffic to paths such that for each $1 \leq i \leq k$ the demand $\gamma_{i}$ is routed over a single path $p \in P^{\left(s, t_{i}\right)}$ without violating the capacity constraints?

The single source unsplittable flow problem is transformed to Problem KPR as follows (see Fig. 8). Add an "aggregated" target $T$. Then, for each $i \in[1, \mathrm{k}]$ add a link $t_{i} \rightarrow T$ with a capacity $\gamma_{i}$.

We shall prove that it is possible to find an assignment of the demands $\gamma_{1}, \gamma_{2}, \ldots, \gamma_{\kappa}$ to paths such that for each $1 \leq \mathrm{i} \leq k$ the demand $\gamma_{i}$ is routed over a single path $p \in P^{\left(s, t_{i}\right)}$ without violating the capacity constraints iff there exists a path flow that transfers $\gamma=\sum_{i=1}^{k} \gamma_{i}$ flow units from $s$ to $T$ over at most $k$ paths without exceeding a network congestion factor of $\alpha=1$. $\Rightarrow$ : For each $1 \leq \mathrm{i} \leq k$, there is exactly one path from $s$ to $t_{i}$ that carries $\gamma_{i}$ flow units without violating the capacity constraints. Hence, there are exactly $k$ paths from $s$ to $T$ that transfers together $\gamma=\sum_{i=1}^{k} \gamma_{i}$ flow units without exceeding a network congestion factor of $\alpha=1$. $\Leftarrow$ : There exists a path flow $f$ that transfers $\gamma=\sum_{i=1}^{k} \gamma_{i}$ flow units from $s$ to $T$ over at most $k$ paths without exceeding a network congestion factor of $\alpha=1$. Consider the cut $\left(A, A^{\prime}\right)$, where $A=V$ and $A^{\prime}=\{T\}$. Since the capacity of the cut $\left(A, A^{\prime}\right)$ is equal to the demand $\gamma$, the path flow $f$ employs each link $t_{i} \rightarrow T$, where $1 \leq i \leq k$. Yet, since it employs at most $k$ paths, $f$ must have exactly one path with positive flow between $s$ and $t_{i}$ for each $1 \leq i \leq k$; moreover, since the flow over each link $t_{i} \rightarrow T$ must equal to the capacity of that link, it holds for each $1 \leq i \leq k$ that the (single) path that $f$ employs in order to carry traffic from $s$ to $t_{i}$ transfers $\gamma_{i}$ flow units.

Thus, Problem KPR is NP-hard.

Although Problem KPR is NP-hard in the general case, we now show that it admits a polynomial solution when the restriction on the number of paths is larger than the number of links (i.e., $K \geq M$ ). Specifically, in the Appendix, we show that it is possible to obtain a flow that minimizes the network congestion factor with a single execution of a max-flow algorithm. Moreover, using the flow decomposition algorithm [12], it is possible to transform in polynomial time every link flow representation into a path flow representation that admits at most $M$ routing paths. Therefore, with a single execution of a max-flow algorithm followed by a single execution of the flow decomposition 
algorithm, it is possible to solve Problem KPR in polynomial time in the case $K \geq M$.

\section{B. Integral Routing Problem}

Our approximation scheme (for the case $K<M$ ) is based on solving an auxiliary problem that minimizes congestion while restricting the flow along each path to be integral in $\gamma / K$. In order to formulate the corresponding problem, consider first the following definition.

Definition 1: Given a network $G(V, E)$, a capacity $c_{e}>0$ for each link $e \in E$, a demand $\gamma$ and an integer $K<M$, a feasible path flow $f$ is said to be $\gamma / K$-integral, if for each path $p \in P^{(s, t)}$, it holds that $f(p)$ is a multiple of $\gamma \backslash K$.

Problem Integral Routing: Given a network $G(V, E)$, two nodes $s, t \in V$, a capacity $c_{e}>0$ for each link $e \in E$, a demand $\gamma>0$, and an integer $K<M$, find a $\gamma / K$-integral path flow that minimizes the network congestion factor, such that the demand $\gamma$ is satisfied.

The following observation shall be used in order to construct a polynomial solution to the integral routing problem.

Lemma 2: Given an instance $\left\langle G,\{s, t\},\left\{c_{e}\right\}, \gamma, K\right\rangle$ of the integral routing problem, the optimal network congestion factor is included in the set $\bar{\alpha} \triangleq\left\{\frac{i \cdot \gamma}{K \cdot c_{e}} \mid e \in E, i \in[0, K] \cap \mathbb{Z}\right\}$.

Proof: In order to prove the lemma, it is sufficient to show that the set $\bar{\alpha}$ contains the network congestion factor of all $\gamma / K$-integral path flows that transfer $\gamma$ flow units from $s$ to $t$ over $G(V, E)$. Assume that $f$ is one such path flow (i.e., $\gamma / K$-integral path flow that transfers $\gamma$ flow units from $s$ to $t$ ), and denote by $\alpha$ its network congestion factor. In order to prove the Theorem, we now show that $\alpha \in \bar{\alpha}$. Since $f$ is a $\gamma / K$-integral path flow it holds by definition that $f_{p}$ is a multiple of $\gamma / K$ for each path $p \in P^{(s, t)}$. Hence, the flow over each link $e \in E$ must also be a multiple of $\gamma / K$, i.e., for each $e \in E$ there exists an integer $i$ in the range $[0, K]$ such that $f_{e}=\frac{i \cdot \gamma}{K}$; therefore, for each $e \in E$ it holds that $\frac{f_{e}}{c_{e}} \in\left\{\frac{i \cdot \gamma}{K \cdot c_{e}} \mid i \in[0, K] \cap \mathbb{Z}\right\}$. In particular, $\max _{e \in E}\left\{\frac{f_{e}}{c_{e}}\right\} \in \bigcup_{e \in E}\left\{\frac{i \cdot \gamma}{K \cdot c_{e}} \mid i \in[0, K] \cap \mathbb{Z}\right\}$ $=\left\{\frac{i \cdot \gamma}{K \cdot c_{e}} \mid e \in E, i \in[0, K] \cap \mathbb{Z}\right\}=\bar{\alpha}$; hence, $\alpha \in \bar{\alpha}$.

Remark 4: Observe that the size of $\bar{\alpha}$ is polynomial in the network size, i.e., $|\bar{\alpha}| \leq M \cdot(K+1)=O\left(M^{2}\right)$.

We now introduce procedure test (Fig. 9), which is given an instance $\left\langle G,\{s, t\},\left\{c_{e}\right\}, \gamma, K\right\rangle$ of the integral routing problem and a restriction $\alpha$ on the network congestion factor. If there exists a $\gamma / K$-integral path flow for the given instance with a network congestion factor of at most $\alpha$, then the procedure returns it (and is said to succeed). Otherwise, the procedure returns Fail.

We turn to explain the main idea behind procedure test. Initially, the procedure multiplies all link capacities by a factor of $\alpha$ in order to impose the restriction on the network congestion factor; indeed, multiplying all capacities by $\alpha$ assures that the flow $f_{e}$ along each link $e \in E$ is at most $\alpha \cdot c_{e}$; therefore, the link congestion factor $\frac{f_{e}}{c_{e}}$ for each $e \in E$, and, thus, the network congestion factor $\max _{e \in E}\left\{\frac{f_{e}}{c_{e}}\right\}$, are at most $\alpha$. Next, the procedure rounds down the capacity of each link to the nearest multiple of $\gamma / K$; since the flow over each path in every solution to the integral routing problem is $\gamma / K$-integral, such a rounding has no effect on the capability to transfer the flow demand $\gamma$. Finally, the procedure applies any standard maximum
Próceúure Test $\left.\left(G,{ }_{\{} s, t\right\},\left\{c_{e}\right\}, \gamma, K, \alpha\right)$

1. Multiply all link capacities by $\alpha$ and round down each capacity to the nearest multiple of $\frac{\gamma}{K}$ i.e., set $\tilde{c_{e}} \leftarrow \frac{\gamma}{K} \cdot\left\lfloor\frac{\alpha \cdot \mathscr{c}_{e}}{\gamma / K}\right\rfloor$ for each $e \in E$.

2. Solve the instance $\left\langle G,(s, t),\left\{\tilde{c}_{e}\right\}\right\rangle$ of the Maximum Flow Problem using the Push Relabel Algorithm [13]. Let the link flow $\left\{f_{e}\right\}$ represent the solution, and let $F$ be the total transferred flow from $s$ to $t$.

3. If $F \geq \gamma$ Else Return the link flow $\left\{f_{e}\right\}$. Return Fail.

Fig. 9. Procedure test.

flow algorithm that returns an integral link flow when all capacities are integral. Since all capacities are $\gamma / K$-integral, the maximum flow algorithm determines a $\gamma / K$-integral link flow that transfers the maximum amount of flow without violating the restriction $\alpha$ on the network congestion factor. If this link flow succeeds to transfer at least $\gamma$ flow units from $s$ to $t$, then the procedure returns it. Otherwise, the procedure fails.

Theorem 4: Given an instance $\left\langle G,\{s, t\},\left\{c_{e}\right\}, \gamma, K\right\rangle$ of the integral routing problem and a restriction $\alpha$ in the network congestion factor, denote by $\alpha^{*}$ the corresponding optimal network congestion factor. Then, procedure test succeeds for the instance $\left\langle G,\{s, t\},\left\{c_{e}\right\}, \gamma, K\right\rangle$ with the restriction $\alpha$ iff $\alpha=\alpha^{*}$.

The proof appears in [4].

Theorem 4 has two important implications that enable to construct an efficient solution to the integral routing problem. First, the theorem establishes that the smallest $\alpha$ for which procedure test succeeds with the input $\left\langle G,\{s, t\},\left\{c_{e}\right\}, \gamma, K, \alpha\right\rangle$ is equal to the optimal network congestion factor $\alpha^{*}$. Therefore, if $S$ is a finite set that includes $\alpha^{*}$ and $\alpha$ is the smallest network congestion factor in $S$ such that procedure test succeeds for the input $\left\langle G,\{s, t\},\left\{c_{e}\right\}, \gamma, K, \alpha\right\rangle$, then $\alpha=\alpha^{*}$. This fact, together with the fact that the set $\bar{\alpha}$ includes $\alpha^{*}$ (as per Lemma 2), imply that, for every instance $\left\langle G,\{s, t\},\left\{c_{e}\right\}, \gamma, K\right\rangle$ of problem integral routing, the optimal network congestion factor $\alpha^{*}$ is the smallest $\alpha \in \bar{\alpha}$, such that procedure test succeeds, for the input $\left\langle G,\{s, t\},\left\{c_{e}\right\}, \gamma, K, \alpha\right\rangle$. Moreover, since in the case of a success procedure test returns the corresponding link flow, finding the smallest $\alpha \in \bar{\alpha}$, such that procedure test succeeds, identifies a link flow with a network congestion factor of $\alpha^{*}$.

The second implication of Theorem 4 enables to employ a binary search when we seek the smallest $\alpha \in \bar{\alpha}$ such that procedure test succeeds. Indeed, it follows from Theorem 4 that, when procedure test succeeds for $\alpha_{1} \in \bar{\alpha}$, it succeeds for all $\alpha \in \bar{\alpha}, \alpha \geq \alpha_{1}$; and when it fails for $\alpha_{2} \in \bar{\alpha}$, it fails for all $\alpha \in \bar{\alpha}, \alpha \leq \alpha_{2}$; thus, if procedure test succeeds for $\alpha_{1} \in \bar{\alpha}$ (alternatively, fails for $\alpha_{2} \in \bar{\alpha}$ ) it is possible to eliminate from further consideration all the elements of $\bar{\alpha}$ that are larger than $\alpha_{1}$ (correspondingly, smaller than $\alpha_{2}$ ). 


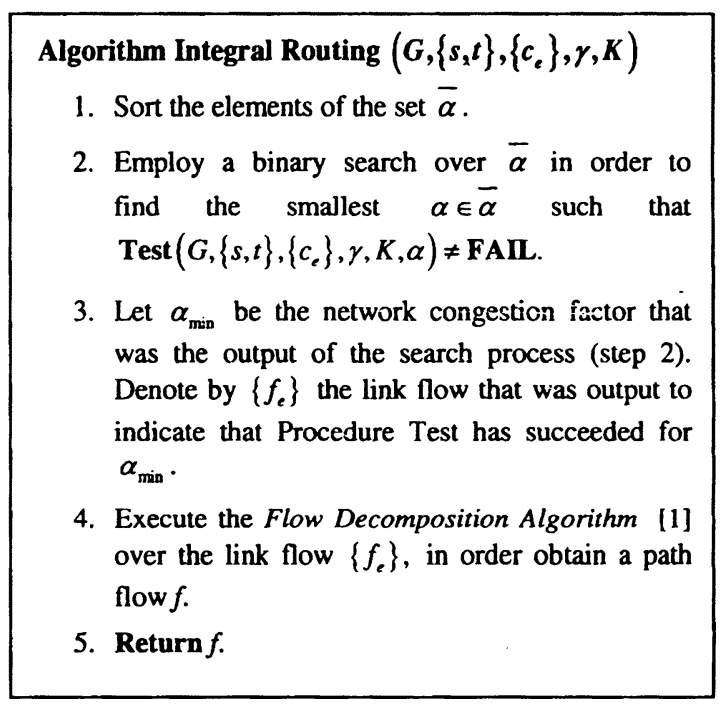

Fig. 10. Algorithm integral routing.

Remark 5: Note that performing a binary search over $\bar{\alpha}$ requires sorting all the elements of $\bar{\alpha}$, which consumes $O(|\bar{\alpha}| \cdot \log |\bar{\alpha}|) \leq O\left(M^{2} \cdot \log N\right)$ operations [10].

Thus, we conclude that the employment of a binary search so as to find the smallest $\alpha \in \bar{\alpha}$ for which procedure test succeeds, establishes a link flow that has the minimum network congestion factor. The optimal solution is then achieved by decomposing the resulting link flow into a path flow via the flow decomposition algorithm [1]. Algorithm integral routing, presented in Fig. 10, specifies these steps.

Our previous discussion is summarized by the following theorem, which establishes that algorithm integral routing solves problem integral routing.

Theorem 5: Given an instance $\left\langle G,\{s, t\},\left\{c_{e}\right\}, \gamma, K\right\rangle$ of problem integral routing, algorithm integral routing returns a $\gamma / K$-integral path flow that transfers at least $\gamma$ flow units from $s$ to $t$, such that the network congestion factor is minimized.

Proof: Consider algorithm integral routing (Fig. 10). By construction, the value $\alpha_{\min }$, identified in step (3) of the algorithm satisfies $\operatorname{Test}\left(G,\{s, t\},\left\{c_{e}\right\}, \gamma, K, \alpha_{\min }\right) \neq$ FAIL. Hence, by the construction of procedure test, the link flow that procedure test returns for the success with $\alpha_{\min }$ transfers $\gamma$ flow units from $s$ to $t$; moreover, since we have shown in the Proof of Theorem 4 that this link flow is $\gamma / K$-integral, the flow decomposition algorithm of step 4 decomposes it into a $\gamma / K$-integral path flow.

It remains to be shown that $\alpha_{\min }$ is the optimal network congestion factor for the given instance. However, this is obvious, since $\alpha_{\min }$ is defined to be the smallest $\alpha \in \bar{\alpha}$ such that $\operatorname{Test}\left(G,\{s, t\},\left\{c_{e}\right\}, \gamma, K, \alpha\right) \neq$ FAIL. Therefore, it follows from Theorem 4 that $\alpha_{\min }$ equals to the smallest $\alpha \in \bar{\alpha}$ such that $\alpha \geq \alpha^{*}$, where $\alpha^{*}$ is the optimal network congestion factor of the given instance. However, since we established in Lemma 2 that $\alpha^{*} \in \bar{\alpha}$, it follows that $\alpha_{\min }=\alpha^{*}$.

We now establish the computational complexity of algorithm integral routing. As was shown in Remark 5, step 1 of the algorithm consumes $O\left(M^{2} \cdot \log N\right)$ operations. Step 2 consumes $O(T(N, M) \cdot \log |\bar{\alpha}|)=O(T(N, M) \cdot \log N)$, where $T(N, M)$ is the computational complexity of the push relabel algorithm that procedure test employs for an $N$-node, $M$-link network. Step 3 consumes $\mathrm{O}(1)$ operations. The flow decomposition algorithm executed in step 4 consumes $\mathrm{O}(N \cdot(M+N))$ operations [1]. Finally, since the representation of a path flow $f$ consists of $O(M \cdot N \cdot \log N)$ bits, ${ }^{1}$ step 5 consumes $O(M \cdot N \cdot \log N)$ operations. Thus, we conclude that, since $G(V, E)$ is connected (i.e., $M \geq N-1)$, the overall complexity of algorithm integral routing is $O\left(M^{2} \cdot \log N+T(N, M) \cdot \log N\right)$. We note that the push relabel algorithm has an implementation with a running time of $O(M \cdot N \cdot \log N)$ [13]. With this implementation, the complexity of algorithm integral routing is $O(M \cdot \log N \cdot(M+N \cdot \log N))$.

\section{Two-Approximation Scheme for Problem KPR}

Finally, we are ready to establish a solution for Problem KPR. To that end, we show that the solution of the integral routing problem can be used in order to establish a constant approximation scheme for Problem KPR. The approximation scheme is based on the following key observation, which links between the optimal solution of problem integral routing and the optimal solution of Problem KPR.

Theorem 6: Consider a network $G(V, E)$ and a demand of $\gamma$ flow units that has to be routed from $s$ to $t$. If $f_{1}$ is a $\gamma / K$-integral path flow that has the minimum network congestion factor and $f_{2}$ is a path flow that minimizes its network congestion factor while routing along at most $K$ paths, then the network congestion factor of $f_{1}$ is at most twice the network congestion factor of $f_{2}$.

Proof: Suppose that $f_{1}$ and $f_{2}$ satisfy the assumptions of the Theorem. Let $\alpha_{1}$ and $\alpha_{2}$ denote the network congestion factor of path flows $f_{1}$ and $f_{2}$, respectively. We have to show that $\alpha_{1} \leq 2 \cdot \alpha_{2}$.

Out of the path flow $f_{2}$, we construct a $\gamma / K$-integral path flow that ships at least $\gamma$ flow units from $s$ to $t$ and has a network congestion factor of at most $2 \cdot \alpha_{2}$. Clearly, such a construction implies that the network congestion factor of every optimal $\gamma / K$-integral path flow that ships $\gamma$ flow units from $s$ to $t$ is at most $2 \cdot \alpha_{2}$; in particular, since $f_{1}$ is one such optimal $\gamma / K$-integral path flow, such a construction establishes that $\alpha_{1} \leq 2 \cdot \alpha_{2}$.

With this goal in mind, define the following construction. First, double the flow along each routing path that $f_{2}$ employs; obviously, the resulting path flow transfers $2 \cdot \gamma$ flow units from $s$ to $t$ along at most $K$ routing paths while yielding a network congestion factor of $2 \cdot \alpha_{2}$. Then, round down the (doubled) flow along each routing path to the nearest multiple of $\gamma / K$; in this process, the flow along each path is reduced by at most $\gamma / K$ flow units. Hence, since there are no more than $K$ routing paths, the total flow from $s$ to $t$ is reduced by at most $\gamma$ units; therefore, since before the rounding operation exactly $2 \cdot \gamma$ flow units were shipped from $s$ to $t$, it follows that after the rounding is performed, the resulting path flow transfers at least $\gamma$ flow units from $s$ to $t$.

$1 f$ consists of $O(M)$ routing paths, each path consists of $O(N)$ links, and each link is represented by $O(\log N)$ bits. Therefore, the collection of all pairs $(p, f(p))$ such that $f(p) \neq 0$ consists of $O(M \cdot N \cdot \log N)$ bits. 


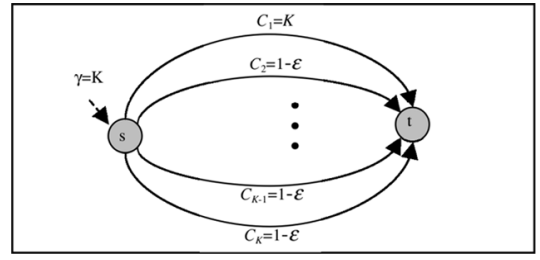

Fig. 11. Tight performance guarantees for algorithm integral routing.

Thus, we have identified a $\gamma / K$-integral path flow that transfers at least $\gamma$ flow units from $s$ to $t$. In addition, since prior to the rounding operation the network congestion factor is $2 \cdot \alpha_{2}$ and the rounding can only reduce flow, the network congestion factor of the constructed path flow is at most $2 \cdot \alpha_{2}$.

Note that, given a network $G(V, E)$ and a demand $\gamma$ that needs to be routed over at most $K$ paths, every $\gamma / K$-integral path flow satisfies the requirement to ship the demand $\gamma$ on at most $K$ different paths. On the other hand, it has been established in Theorem 6 that the network congestion factor obtained by an optimal $\gamma / K$-integral path flow is at most twice the network congestion factor of an optimal flow that admits at most $K$ routing paths. Thus, computing a $\gamma / K$-integral path flow that has the minimum network congestion factor satisfies the restriction on the number of routing paths and obtains a network congestion factor that is at most twice larger than the optimum. We summarize the previous discussion in the following corollary, which yields an approximation scheme for Problem KPR.

Corollary 1: Consider a network $G(V, E)$, a demand $\gamma$, and a restriction on the number of routing paths $K$. The employment of algorithm integral routing for the establishment of a $\gamma / K$-integral path flow that minimizes the network congestion factor provides a two-approximation scheme for Problem KPR with a complexity of $O(M \cdot \log N \cdot(M+N \cdot \log N))$.

Finally, we note that the performance guarantee obtained in Corollary 1 for algorithm integral routing is tight, i.e., there are instances for which the network congestion factor obtained by algorithm integral routing is asymptotically twice the optimal network congestion factor. The following example presents one such instance.

Example 2: Consider the $K$ parallel-links network in Fig. 11. Assume that the upper link has a capacity $K$ and each other link has a capacity $1-\varepsilon$ for some small value $\varepsilon>0$. Furthermore, assume that the flow demand $\gamma$ is equal to $K$ and the maximum number of paths over which traffic can be assigned is also $K$. Consider first the solution obtained by algorithm integral routing. The algorithm first rounds all capacities down to the nearest multiple of $\frac{\gamma}{K}$, namely, 1 ; hence, all link capacities are zeroed except for the capacity on the upper link that remains to be equal to $K$. Accordingly, the solution returned by algorithm integral routing assigns $K$ flow units over the upper link, hence, resulting with a network congestion factor of 1 . On the other hand, it is easy to see that for a sufficiently small value of $\varepsilon$, a solution that assigns $0.5 \cdot K$ flow units to the upper link and $\frac{0.5 \cdot K}{K-1}$ flow units to each other link is feasible and has a network congestion factor of $\frac{0.5 \cdot K}{K-1} \cdot \frac{1}{1-\varepsilon}$. Thus, the ratio between the two network congestion factors is $\frac{1}{\frac{0.5 . K}{K-1} \cdot \frac{1}{1-\varepsilon}} \rightarrow 2$ for $K \rightarrow \infty$ and $\varepsilon \rightarrow 0$.

\section{Simulation Results}

The goal of this section is to demonstrate how much is gained by employing optimal multipath routing algorithms for congestion minimization. To that end, we present a comparison between the congestion obtained by an optimal multipath routing algorithm to the congestion obtained by the popular heuristics ECMP and OMP. Through comprehensive simulations, we show that multipath routing solutions obtained by optimal congestion reduction schemes are fundamentally more efficient. We generated two classes of random networks: Power-law topologies [11] and Waxman topologies [30]. For each class, we generated 10000 networks and conducted the following measurements over each network: 1) the network congestion factor $\alpha_{E}$ produced by invoking ECMP; 2) the network congestion factor $\alpha_{O}$ produced by invoking OMP; and 3) the network congestion factor $\alpha^{*}(L)$ produced by an optimal assignment of traffic to paths with a length of at most $L, L \in\left\{L^{*}, 1.1 \cdot L^{*}, 1.2 \cdot L^{*}, 1.3 \cdot L^{*}, 1.4 \cdot L^{*}\right.$, $\left.1.5 \cdot L^{*}, 1.6 \cdot L^{*}, 1.7 \cdot L^{*}, 1.8 \cdot L^{*}, 1.9 \cdot L^{*}, 2 \cdot L^{*}\right\}$, where $L^{*}$ is the length of a shortest path. In all runs, we assumed that the link capacities are uniformly distributed in $[5,150] \mathrm{MB} / \mathrm{s}$, the bandwidth requests is uniformly distributed in $[1,5] \mathrm{MB} / \mathrm{s}$, and the length of each link is uniformly distributed in $[1,50]$. The following definition is used to evaluate the gain in the use of optimal multipath routing algorithms.

Definition 2: Given a network $G(V, E)$ a pair of nodes $s, t \in$ $V$, a demand $\gamma$, and a length restriction $L$, define by $\rho_{E}(L)$ the ratio between the optimal network congestion factor and that obtained by ECMP; similarly, define by $\rho_{O}(L)$ the ratio between the optimal network congestion factor and that obtained by OMP, i.e.,

$$
\rho_{E}(L) \triangleq \frac{\alpha^{*}(L)}{\alpha_{E}}, \quad \rho_{O}(L) \triangleq \frac{\alpha^{*}(L)}{\alpha_{O}} .
$$

We proceed to specify the way we generated each class of topologies, starting with Waxman topologies. Our construction follows the lines of [30]. We first located the source and the destination at the diagonally opposite corners of a square area of unit dimension. Then, we randomly spread 198 nodes over the square. Finally, we introduced a link between each two nodes $u$ and $v$, with the following probability, which depended on the distance between them, $\delta(u, v)$ :

$$
p(u, v)=\alpha \cdot \exp \left[\frac{-\delta(u, v)}{\beta \cdot \sqrt{2}}\right]
$$

using $\alpha=2$ and $\beta=0.2$. The previous approach resulted in 200 nodes and approximately 1800 links per network topology.

We now refer to the way we generated power-law topologies. Our construction followed the lines of [11]. First, we randomly assigned a certain number of out-degree credits to each node, using the power-law distribution $\beta \cdot x^{-\alpha}$, where $\alpha=0.3$ and $\beta=3$. Then, we connected the nodes so that every node obtained the assigned out degree. More specifically, we randomly picked a pair of nodes $u$ and $v$, and assigned a directed link from $u$ to $v$ if $u$ had some remaining out-degree credits and link $u \rightarrow v$ had not been defined already. Whenever a link $u \rightarrow v$ was placed between the corresponding nodes, we also decremented the out-degree credit of node $u$. When the selected pair 


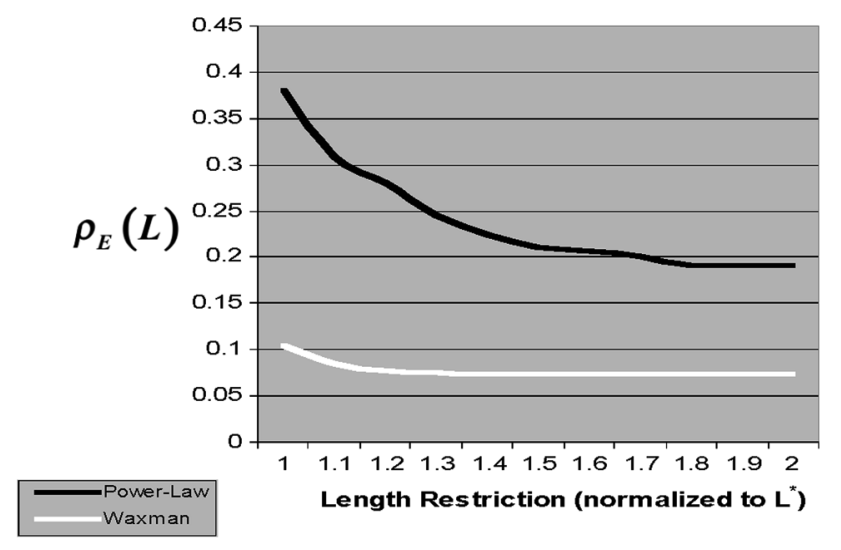

Fig. 12. Ratio between the network congestion produced by an optimal multipath routing assignment and the network congestion produced by ECMP.

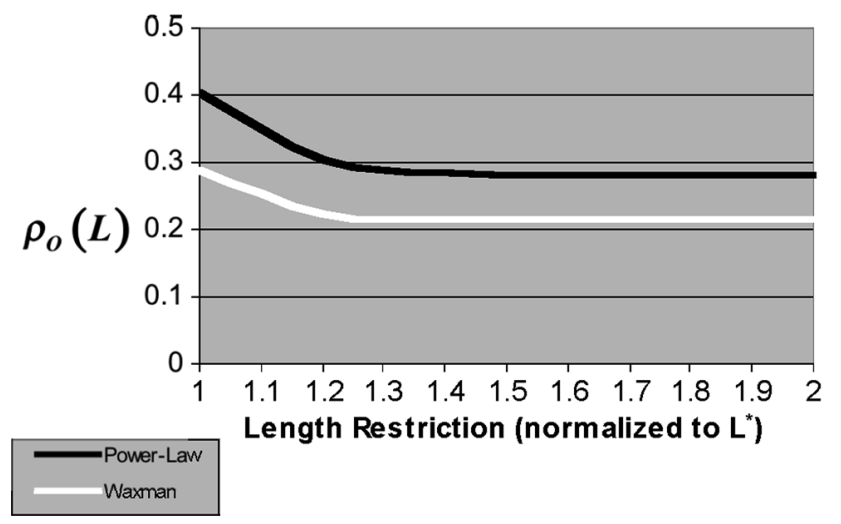

Fig. 13. Ratio between the network congestion produced by an optimal multipath routing assignment and the network congestion produced by OMP.

of nodes was not suitable for a link, we continued to pick pairs of nodes until finding one that was suitable. The previous strategy resulted in 200 nodes and approximately 1200 links per network topology.

Our results are summarized in Figs. 12 and 13. Note that, for power-law topologies, if the ECMP or OMP heuristics had an optimal mechanism to distribute traffic among the shortest paths, the network congestion factor would have been reduced by a factor of at least 2.5; moreover, for Waxman topologies, this factor of improvement is greater than 3 for OMP and greater than 9.5 for ECMP. Next, observe that optimal traffic distribution mechanisms that allow relaxing the requirement to route along shortest paths by just 20\% produce a network congestion factor that, in power-law topologies, is at least 3.3 times smaller than with OMP and ECMP; moreover, for Waxman topologies, this factor of improvement is more than 4.5 for OMP and is more than 12 for ECMP. Thus, for $L \approx 1.2 \cdot L^{*}$, Algorithm RMP or its $\varepsilon$-optimal approximation can drastically reduce network congestion at the price of routing along paths that are just slightly longer than the shortest.

\section{CONCLUSION}

Previous multipath routing schemes for congestion avoidance focused on heuristic methods. Yet, our simulations indicate that optimal congestion reduction schemes are significantly more efficient. Accordingly, we investigated multipath routing as an optimization problem of minimizing network congestion and considered two fundamental problems. Although both have been shown to be computationally intractable, they have been found to admit efficient approximation schemes. Indeed, for each problem, we have established a polynomial time algorithm that approximates the optimal solution by a (small) constant approximation factor.

A common feature that both approximations share is the discretization of the set of feasible solutions. Whereas the solution to Problem KPR is established by restricting the flow along each path to be integral in $\gamma / K$, the solution to Problem RMP is established by restricting all lengths to be integral in some common scaling factor. These discretizations enable to reduce the space of feasible solutions and therefore obtain polynomial running time algorithms.

While this study has laid the algorithmic foundations of two fundamental multipath routing problems, there are still many challenges to overcome. One major challenge is to establish an efficient unifying scheme that combines the two problems. Furthermore, it is interesting to consider the distributed implementation of our solutions. Since algorithm integral routing (that is used to solve Problem KPR) invokes a set of successive computations of a max-flow algorithm, its distributed implementation is straightforward due to [3] that provides distributed implementations for max-flow algorithms. The distributed implementation of Algorithm RMP remains an open issue for future investigation. Finally, as discussed in [4], multipath routing offers a rich ground for research also in other contexts, such as survivability, recovery, network security, and energy efficiency. We are currently working on these issues and have obtained several results regarding survivability [5].

\section{REFERENCES}

[1] R. K. Ahuja, T. L. Magnanti, and J. B. Orlin, Network Flows: Theory, Algorithm, and Applications. Englewood Cliffs, NJ: Prentice-Hall, 1993.

[2] D. Awduche, J. Malcolm, J. Agogbua, M. O’Dell, and J. McManus, "Requirements for traffic engineering over MPLS," IETF RFC 2702, Sep. 1999.

[3] B. Awerbuch, "Reducing complexities in the distributed max-flow and breadth-first-search algorithms by means of network synchronization," Network, vol. 15, pp. 425-437, 1985.

[4] R. Banner and A. Orda, "Multipath routing algorithms for congestion minimization,” Dept. Electr. Eng., Technion, Haifa, Israel, CCIT Rep. 429, (2005). [Online]. Available: http://www.ee.technion.ac.il/people/ ron/Congestion.pdf

[5] R. Banner and A. Orda, "The power of tuning: A novel approach for the efficient design of survivable networks," in Proc. IEEE ICNP, 2004, pp. 2-11.

[6] D. Bertsekas and R. Gallager, Data Networks. Englewood Cliffs, NJ: Prentice-Hall, 1992.

[7] A. Borodin and R. El Yaniv, Online Computation and Competitive Analysis. Cambridge, U.K.: Cambridge Univ. Press, 1998.

[8] I. Cidon, R. Rom, and Y. Shavitt, "Analysis of multi-path routing," IEEE Trans. Netw., vol. 7, no. 6, pp. 885-896, Dec. 1999.

[9] S. Chen and K. Nahrstedt, "An overview of quality-of-service routing for the next generation high-speed networks: Problems and solutions," IEEE Trans. Netw., vol. 12, no. 6, pp. 64-79, Dec. 1998.

[10] T. Cormen, C. Leiserson, and R. Rivest, Introduction to Algorithms. Cambridge, MA: MIT Press, 2001

[11] M. Faloutsos, P. Faloutsos, and C. Faloutsos, "On power-law relationships of the Internet topology," in Proc. ACM SIGCOMM, 1999, pp. 251-262.

[12] M. R. Garey and D. S. Johnson, Computers and Intractability. San Francisco, CA: Freeman, 1979. 
[13] A. V. Goldberg and R. E. Tarjan, "A new approach to the maximum flow problem," J. ACM, vol. 35, no. 4, pp. 921-940, 1988.

[14] H. Han, S. Shakkottai, C. V. Hollot, R. Srikant, and D. Towsley, "Multipath TCP: A joint congestion control and routing scheme to exploit path diversity in the Internet," IEEE/ACM Trans. Netw., vol. 14, no. 6, pp. 1260-1271, Dec. 2006.

[15] C. Huitema, Routing in the Internet. Englewood Cliffs, NJ: PrenticeHall, 1995.

[16] S. Iyer, S. Bhattacharyya, N. Taft, N. McKeoen, and C. Diot, "A measurement based study of load balancing in an IP backbone," Sprint ATL, Tech. Rep. TR02-ATL-051027, May 2002.

[17] Y. Jia, I. Nikolaidis, and P. Gburzynski, "Multiple path QoS routing," in Proc. ICC, 2001, pp. 2583-2587.

[18] N. Karmarkar, "A new polynomial-time algorithm for linear programming," Combinatorica, vol. 4, pp. 373-395, 1984.

[19] F. P. Kelly, A. K. Maulloo, and D. K. H. Tan, "Rate control in communication networks: Shadow prices, proportional fairness and stability," J. Oper. Res. Soc., vol. 49, pp. 237-252, 1998.

[20] F. P. Kelly and T. Voice, "Stability of end-to-end algorithms for joint routing and rate control," Comput. Commun. Rev., vol. 35, no. 2, pp. $5-12,2005$.

[21] J. Kleinberg, "Single-source unsplittable flow," in Proc. 37th IEEE FOCS, 1996, pp. 68-77.

[22] J. Moy, “OSPF Version 2," IETF RFC 2328, Apr. 1998.

[23] S. Nelakuditi and Z.-L. Zhang, "On selection of paths for multipath routing," in Proc. IWQoS, 2001, pp. 170-186.

[24] V. Paxson, "End-to-end routing behavior in the Internet," in Proc. ACM SIGCOMM, 1996, pp. 25-38.

[25] P. Papadimitratos and Z. J. Haas, "Secure routing for mobile Ad hoc networks," in Proc. CNDS, 2002, pp. 70-82.

[26] E. Rosen, A. Viswanathan, and R. Callon, "Multiprotocol label switching architecture," IETF RFC 3031, 2001.

[27] D. Thaler and C. Hopps, "Multipath issues in unicast and multicast next-hop selection," IETF RFC 2991, 2000.

[28] C. Villamizar, "OSPF optimised multipath (OSPF-OMP)," Internet Draft, 1998

[29] Y. Wang and Z. Wang, "Explicit routing algorithms for Internet traffic engineering," in Proc. ICCN'99, 1999, pp. 582-588.

[30] B. M. Waxman, "Routing of multipoint connections," IEEE J. Sel. Areas Commun., vol. 6, no. 9, pp. 1617-1622, Dec. 1988.
[31] A. E. I. Widjaja, "Mate: MPLS adaptive traffic engineering," Internet Draft, Aug. 1998.

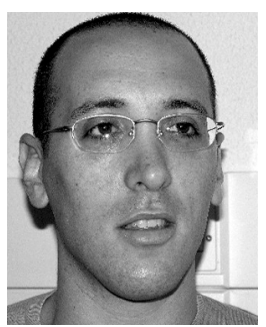

Ron Banner (S'04) received the B.Sc. degree in computer engineering from the Technion-Israel Institute of Technology, Haifa, Israel, in 2001, where he is currently pursuing the $\mathrm{Ph} . \mathrm{D}$. degree in electrical engineering.

His current research interests include network routing and survivability.

Mr. Banner is a recipient of the Gutwirth Award for Special Excellence in M.Sc. Studies in 2003, the Prof. Andrew Viterbi Fellowship for Outstanding Graduate Students in 2004, the ICNP'2004 Best Paper Award, the Jury Award for Outstanding Graduate Students in Electrical Engineering in 2005, and the Gutwirth Award for Special Excellence in Ph.D. studies in 2005.

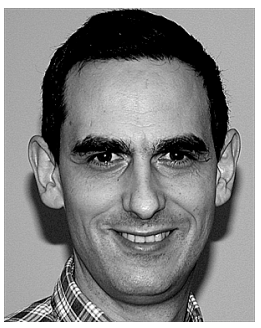

Ariel Orda (S'84-M'92-SM'97-F'06) received the B.Sc. (summa cum laude), M.Sc., and D.Sc. degrees in electrical engineering from the Technion-Israel Institute of Technology, Haifa, Israel, in 1983, 1985, and 1991, respectively.

Since 1994, he has been with the Department of Electrical Engineering, Technion. His current research interests include network routing, survivability, QoS provisioning, wireless networks, the application of game theory to computer networking, and network pricing.

Dr. Orda is a recipient of the Award of the Chief Scientist in the Ministry of Communication in Israel, a Gutwirth Award for Outstanding Distinction, the Research Award of the Association of Computer and Electronic Industries in Israel, the Jacknow Award for Excellence in Teaching, an ICNP'2004 Best Paper Award, and the 2005 Klein Award for Excellence in Research. He served as Program co-chair of IEEE Infocom'2002 and is an Editor of the IEEE/ACM TRANSACTIONS ON NETWORKING and of the Journal of Computer Networks. 